\title{
Opportunistic Interference Alignment with 1-Bit Feedback in 3-Cell Interference Channels
}

\author{
Zhinan $\mathrm{Xu}^{1}$, Mingming $\mathrm{Gan}^{1}$, Thomas Zemen ${ }^{1,2}$ \\ ${ }^{1}$ FTW (Telecommunications Research Center Vienna), Vienna, Austria \\ ${ }^{2}$ AIT Austrian Institute of Technology, Vienna, Austria \\ Contact: xu@ftw.at
}

\begin{abstract}
Opportunistic interference alignment (OIA) exploits channel randomness and multiuser diversity by user selection. The transmitter needs channel state information (CSI), which is usually measured on the receiver side and sent to the transmitter side via a feedback channel. Lee and Choi show that $d$ degrees of freedom (DoF) per transmitter are achievable in a 3-cell MIMO interference channel assuming a fully informed network, where every user feeds back a real-valued variable to their own transmitter. This paper investigates the achievable DoF using only 1-bit feedback per user. We prove that 1-bit feedback is sufficient to achieve the optimal DoF $d$. Most importantly, the required number of users for OIA with 1-bit feedback remains the same as with real-valued feedback. Moreover, for a given system configuration, we provide an optimal choice of the 1-bit quantizer, which captures most of the capacity provided by a system with real-valued feedback.
\end{abstract}

\section{INTRODUCTION}

Interference is a crucial limitation in next generation cellular systems. To address this problem, interference alignment (IA) has attracted much attention and has been extensively studied lately. IA is able to achieve the optimal degrees of freedom (DoF) at high signal-to-noise ratios (SNR) resulting in a rate of $M / 2 \cdot \log (\mathrm{SNR})+o(\log (\mathrm{SNR}))$ for the $M$ cell interference channel. For IA, a closed-form solution of the precoding vectors for single antenna nodes with symbol extension is known [1]. However, this coding scheme is based on the assumption that global channel state information (CSI) is available at all nodes, which is extremely hard to achieve and maybe even impossible.

For the sake of complexity reduction, opportunistic interference alignment (OIA) has been studied lately [2]-[7]. The key idea of OIA is to exploit the channel randomness and multiuser diversity by proper user selection. In [2]-[7], signal subspace dimensions are used to align the interference signals. Each transmitter opportunistically selects and serves the user whose interference channels are most aligned to each other. The degree of alignment is quantified by a metric. To facilitate a user selection algorithm, all potential users associated with the transmitter are required to calculate and feedback the metric value based on the local CSI. Perfect IA can be achieved asymptotically if the number of users scales fast enough with SNR. The corresponding user scaling law to obtain the optimal DoF is characterized for multiple access channels in [2], [3] and for downlink interference channels in [5]-[7].

The work in [5] decouples a multiple-input multiple-output (MIMO) interference channel into multiple SIMO interference channels and guarantees one spatial stream for each selected user. Since each stream is associated to one metric value, multiple metric values have to be fed back to allow for multiple streams at the transmitters. The work of [6] reduces the number of users to achieve the optimal DoF at the expense of increased feedback information from each user. In [6], each user has to feed back a metric value and a channel vector to cancel intra-cell interference. To enable multiple spatial streams for each selected user, the authors of [7] investigate the required user scaling in 3-cell MIMO interference channels and show that the optimal DoF $d$ is achieved if the number of users $K$ is scaled as $K \propto \mathrm{SNR}^{d^{2}}$. Therefore, at higher SNR, a larger number of users is required to achieve the optimal DoF. Clearly, the level of required total CSI feedback also increases proportionally to the number of users. However, in practical systems, the feedback is costly and the bandwidth of the feedback channel is limited. As a result, the feedback rate should be kept as small as possible.

For opportunistic transmission in point-to-point systems, the problem of feedback reduction is tackled in [8]-[10] by selective feedback. The solution is to let the users threshold their receive SNRs and notify the transmitter only if their SNRs exceed a predetermined threshold. The work in [8], [9] reduces the number of real-valued variables that must be fed back to the transmitter in SISO and MIMO multiuser channels respectively. But [8], [9] do not directly address the question of feedback rate since transmission of real-valued variables requires infinite rate. The work in [10] investigates the performance of opportunistic multiuser systems using limited feedback and proves that 1-bit feedback per user can capture a double-logarithmic capacity growth with the number of users. Note that [8]-[10] consider interference-free pointto-point transmissions.

Unlike point-to-point systems where the imperfect CSI causes only an SNR offset in the capacity, the accuracy of the CSI in interference channels affects the slope of the rate curve, i.e., the DOF. Thus, for OIA, a relation to the DoF using selective feedback is critical. Can we reduce the amount of feedback and still preserve the optimal DoF? This is addressed in our paper [11] using real-valued feedback. It shows that the amount of feedback can be dramatically reduced by more than one order of magnitude while still preserving the essential DoF promised by conventional OIA with full feedback. However, to the best of our knowledge, the achievability of the optimal 


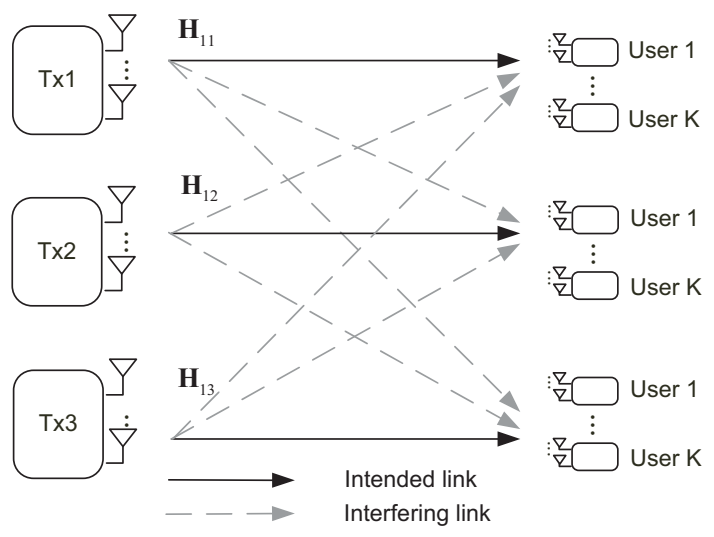

Fig. 1. Three-cell MIMO interference channel with $K$ candidates in each cell

DoF with limited feedback is still unknown. ${ }^{1}$ In this paper, we address this problem by 1-bit feedback for 3-cell MIMO interference channels.

\section{Contributions of this paper:}

- We prove that the feedback of only 1 bit per user is sufficient to achieve the full DoF (without requiring more users) if the 1-bit quantizer is chosen judiciously.

- We derive the scheduling outage probability according to the metric distribution for 1-bit feedback.

- We provide an optimal choice of the 1-bit quantizer to achieve the DoF of 1, which captures most of the capacity provided by a system with full feedback. To achieve a $\mathrm{DoF}>1$, an asymptotic threshold choice is given.

- We generalize the design of the DoF achievable threshold choices and provide the mathematical expression.

Notations: We denote a scalar by $a$, a column vector by a and a matrix by $\mathbf{A}$. The superscript ${ }^{\mathrm{T}}$ and ${ }^{\mathrm{H}}$ stand for transpose and Hermitian transpose, respectively. $\mathbb{E}[\cdot]$ denotes the expectation operation. $\mathbf{I}_{N}$ is the $N \times N$ identity matrix. For a given function $f(N)$, we write $g(N)=O(f(N))$ if and only if $\lim _{N \rightarrow \infty}|g(N) / f(N)|$ is bounded.

\section{SYSTEM MODEL}

Let us consider the system model for the 3-cell MIMO interference channel, as shown in Fig. 1. It consists of 3 transmitters with $N_{\mathrm{T}}$ antennas, each serving $K$ users with $N_{\mathrm{R}}$ antennas. The channel matrix from transmitter $j$ to receiver $k$ in cell $i$ is denoted by $\mathbf{H}_{i, j}^{k} \in \mathbb{C}^{N_{\mathrm{R}} \times N_{\mathrm{T}}}, \forall i, j \in\{1,2,3\}$ and $k \in\{1, \ldots, K\}$. Every element of $\mathbf{H}_{i, j}^{k}$ is assumed as an independent identically distributed (i.i.d.) symmetric complex Gaussian random variable with zero mean and unit variance.

For a given transmitter, its signal is only intended to be received and decoded by a single user for a given signaling interval. The signal received at receiver $k \in\{1, \ldots, K\}$ in cell $i$ at a given time instant is the superposition of the signals

\footnotetext{
${ }^{1}$ We are interested in limited feedback for the metric value. The work of [6] addresses limited feedback to quantize a channel vector, which is not relevant to our work
}

transmitted by all three transmitters, which can be written as

$$
\mathbf{x}_{i}^{k}=\mathbf{H}_{i, i}^{k} \mathbf{s}_{i}+\sum_{j=1, j \neq i}^{3} \mathbf{H}_{i, j}^{k} \mathbf{s}_{j}+\mathbf{n}_{i}^{k},
$$

where vector $\mathbf{s}_{j} \in \mathbb{C}^{d \times 1}$ denotes $d$ transmitted symbols from transmitter $j$ with power constraint $\mathbb{E}\left\{\mathbf{s}_{j} \mathbf{s}_{j}^{\mathrm{H}}\right\}=\frac{P}{d} \mathbf{I}_{d}$. The additive complex symmetric Gaussian noise $\mathbf{n}_{i}^{k} \sim \mathcal{C N}\left(0, \mathbf{I}_{N_{\mathrm{R}}}\right)$ has zero mean and unit variance. Thus, the SNR becomes $\mathrm{SNR}=P$. In this paper, we confine ourselves to the nontrivial case of $N_{\mathrm{R}}=2 d$ and $N_{\mathrm{T}}=d$. This is interesting because it is the minimum setup to achieve the full $\operatorname{DoF} d$ at each receiver. In case the number of receive antennas $N_{\mathrm{R}}>2 d, N_{\mathrm{R}}-2 d$ DoF can be obtained with probability one even without interference management because uncoordinated interference signals will span a subspace with a maximum of $2 d$ dimensions in the space $\mathbb{C}^{N_{\mathrm{R}}}$. On the other hand if $N_{\mathrm{R}}<2 d$, the full DoF $d$ is not achievable because the interference signals will span at least a $d$ dimensional subspace even when they are perfectly aligned.

Defining $\mathbf{U}_{i}^{k} \in \mathbb{C}^{N_{\mathrm{R}} \times d}$ as the postfiltering matrix at receiver $k$ in cell $i$, the received signal of user $k$ in cell $i$ becomes

$$
\begin{aligned}
\mathbf{y}_{i}^{k} & =\mathbf{U}_{i}^{k^{\mathrm{H}}} \mathbf{x}_{i}^{k} \\
& =\mathbf{U}_{i}^{k^{\mathrm{H}}} \mathbf{H}_{i, i}^{k} \mathbf{s}_{i}+\sum_{j=1, j \neq i}^{3} \mathbf{U}_{i}^{k^{\mathrm{H}}} \mathbf{H}_{i, j}^{k} \mathbf{s}_{j}+\overline{\mathbf{n}}_{i}^{k}
\end{aligned}
$$

where $\overline{\mathbf{n}}_{i}^{k}=\mathbf{U}_{i}^{k}{ }^{\mathrm{H}} \mathbf{n}_{i}^{k}$ denotes the effective spatially white noise vector. The achievable instantaneous rate for user $k$ in cell $i$ becomes

$$
\begin{aligned}
R_{i}^{k}=\log _{2} \operatorname{det} & \left(\mathbf{I}_{d}+\frac{P}{d} \mathbf{U}_{i}^{k}{ }^{\mathrm{H}} \mathbf{H}_{i, i}^{k} \mathbf{H}_{i, i}^{k}{ }^{\mathrm{H}} \mathbf{U}_{i}^{k}\right. \\
& \left.\left(\frac{P}{d} \sum_{j=1, j \neq i}^{3} \mathbf{U}_{i}^{k^{\mathrm{H}}} \mathbf{H}_{i, j}^{k} \mathbf{H}_{i, j}^{k}{ }^{\mathrm{H}} \mathbf{U}_{i}^{k}+\mathbf{I}_{d}\right)^{-1}\right) .
\end{aligned}
$$

\section{Conventional OIA}

Without requiring global channel knowledge, OIA is able to achieve the same DoF as IA with only local CSI feedback within a cell. In this section, we describe the selection criteria and the design of the postfilter for the conventional OIA algorithm. The key idea of OIA [7] is to exploit the channel randomness and the multi-user diversity, using the following procedure:

- Each transmitter sends out a reference signal.

- Each user equipment measures the channel quality using a specific metric.

- Every user feeds back the value of the metric to its own transmitter.

- The transmitter selects a user in its own cell for communication according to the feedback values.

The transmitters aim at choosing a user, who observes most aligned interference signals from the other transmitters. The degree of alignment is quantified by a subspace distance measure, named chordal distance. It is generally defined as

$$
d_{\mathrm{c}}(\mathbf{A}, \mathbf{B})=1 / \sqrt{2}\left\|\mathbf{A} \mathbf{A}^{\mathrm{H}}-\mathbf{B B}^{\mathrm{H}}\right\|_{\mathrm{F}}
$$


where $\mathbf{A}, \mathbf{B} \in \mathbb{C}^{N_{\mathrm{R}} \times d}$ are the orthonormal bases of two subspaces and $d_{\mathrm{c}}{ }^{2}(\mathbf{A}, \mathbf{B}) \leq d$. For OIA, each user finds an orthonormal basis $\mathbf{Q}$ of the column space spanned by the two interference channels respectively, i.e., $\mathbf{Q}_{i p}^{k} \in \operatorname{span}\left(\mathbf{H}_{i p}^{k}\right)$ and $\mathbf{Q}_{i q}^{k} \in \operatorname{span}\left(\mathbf{H}_{i q}^{k}\right)$ where $p=(i+1 \bmod 3)$ and $q=(i+2$ mod 3). Then the users calculate the distance between two interference subspaces using the obtained orthonormal basis, yielding

$$
\mathcal{D}_{i}^{k}=d_{\mathrm{c}}^{2}\left(\mathbf{Q}_{i p}^{k}, \mathbf{Q}_{i q}^{k}\right),
$$

where $\mathcal{D}_{i}^{k}$ is the distance measured at user $k$ in cell $i$. For conventional OIA, all users feed back the distance measure to their own transmitter and the user selected by transmitter $i$ is given by

$$
k^{*}=\arg \min _{k} \mathcal{D}_{i}^{k} .
$$

Therefore, the metric value of the selected user becomes $\mathcal{D}_{i}^{k^{*}}$. Defining the received interference covariance matrix of the selected user $k^{*}$ as

$$
\mathbf{R}_{i}^{k^{*}}=\mathbf{H}_{i p}^{k^{*}} \mathbf{H}_{i p}^{k^{* \mathrm{H}}}+\mathbf{H}_{i q}^{k^{*}} \mathbf{H}_{i q}^{k^{* \mathrm{H}}},
$$

the postfilter applied at the selected user becomes

$$
\mathbf{U}_{i}^{k^{*}}=\left[\overrightarrow{\mathbf{u}}_{d+1}\left(\mathbf{R}_{i}^{k^{*}}\right), \cdots, \overrightarrow{\mathbf{u}}_{N_{\mathrm{R}}}\left(\mathbf{R}_{i}^{k^{*}}\right)\right]
$$

where $\overrightarrow{\mathbf{u}}_{n}(\mathbf{R})$ represent the singular vector corresponding to the $n$-th largest singular value of $\mathbf{R}$.

\section{The Achievable DoF of OIA with 1-Bit FEEDBACK}

In this section, we introduce the concept of 1-bit feedback for OIA. The achievability of the DoF is proved for $d=1$ first, where a closed-form solution exists. We generalize the result to all $d>1$ based on asymptotic analysis.

\section{A. Preliminaries of the Chordal Distance Measure}

As shown in [12], for quantizing a source $\mathbf{A}$ arbitrarily distributed on the Grassmannian manifold $\mathcal{G}_{N_{\mathrm{R}}, d}(\mathbb{C})$ using a random codebook $\mathcal{C}_{\text {rnd }}$ with $K$ codewords, the second moment of the chordal distance can be bounded as

$$
\begin{aligned}
Q(K) & =\mathbb{E}\left[\min _{\mathbf{C}_{k} \in \mathcal{C}_{\mathrm{rnd}}} d_{\mathrm{c}}^{2}\left(\mathbf{A}, \mathbf{C}_{k}\right)\right] \\
& \leq \frac{\Gamma\left(\frac{1}{d\left(N_{\mathrm{R}}-d\right)}\right)}{d\left(N_{\mathrm{R}}-d\right)}\left(K c_{N_{\mathrm{R}}, d}\right)^{-\frac{1}{d\left(N_{\mathrm{R}}-d\right)}}
\end{aligned}
$$

where $\Gamma(\cdot)$ denotes the Gamma function and the random codebook $\mathcal{C}_{\text {rnd }} \subset \mathcal{G}_{N_{\mathrm{R}}, d}(\mathbb{C})$. The constant $c_{N_{\mathrm{R}}, d}$ is the ball volume on the Grassmannian manifold $\mathcal{G}_{N_{\mathrm{R}}, d}(\mathbb{C})$, i.e.

$$
c_{N_{\mathrm{R}}, d}=\frac{1}{\Gamma\left(d\left(N_{\mathrm{R}}-d\right)+1\right)} \prod_{i=1}^{d} \frac{\Gamma\left(N_{\mathrm{R}}-i+1\right)}{\Gamma(d-i+1)} .
$$

The problem of selecting the best user out of $K$ users is equivalent to quantizing an arbitrary subspace with $K$ random subspaces on the Grassmannian manifold $\mathcal{G}_{N_{\mathrm{R}}, d}(\mathbb{C})$ [7, Lemma 4]. Therefore, we have $\mathbb{E}\left[\mathcal{D}_{i}^{k}\right]=Q(1)$ and $\mathbb{E}\left[\mathcal{D}_{i}^{k^{*}}\right]=Q(K)$.
We briefly revisit the results obtained in [7], which will be used for comparison with our 1-bit feedback OIA. A finite number of users $K$ results in residual interference. Let us define the rate loss term due to residual interference as

$$
R_{\operatorname{loss}_{i}}^{k}=\log _{2} \operatorname{det}\left(\sum_{j=1, j \neq i}^{3} \mathbf{U}_{i}^{k}{ }^{\mathrm{H}} \overline{\mathbf{H}}_{i, j}^{k} \overline{\mathbf{H}}_{i, j}^{k \mathrm{H}} \mathbf{U}_{i}^{k}+\mathbf{I}_{d}\right) \text {. }
$$

When the cell $i$ has $K$ users, the average rate loss at the selected user $k^{*}$ can be bounded as

$$
\begin{aligned}
\mathbb{E}\left[R_{\mathrm{loss} i}^{k^{*}}\right] & \leq d \cdot \log _{2}\left(1+\frac{P}{d} \cdot \mathbb{E}\left[\mathcal{D}_{i}^{k^{*}}\right]\right) \\
& =d \cdot \log _{2}\left(1+\frac{P}{d} \cdot Q(K)\right),
\end{aligned}
$$

where (13) is obtained due to [7, Lemma 6].

The achievable DoF of transmitter $i$ using OIA can be expressed by $d-\lim _{P \rightarrow \infty} \frac{\mathbb{E}\left[R_{\operatorname{loss}_{i} k^{*}}\right]}{\log _{2} P}$. The DoF $d^{\prime}$ is achieved if the number of users is scaled as [7, Theorem 2]

$$
K \propto P^{d d^{\prime}} \text {. }
$$

\section{B. One-Bit Feedback by Thresholding}

For conventional OIA, the user selected for transmission is the one with the smallest chordal distance measure. This requires that the transmitter collects the perfect real-valued chordal distance measures from all the users. However, the feedback of real values require infinite bandwidth. The question of how to efficiently feedback the required CSI is still not solved for OIA. To address this problem, we propose a threshold-based 1-bit feedback strategy where each user compares the locally measured chordal distance to a predefined threshold $x_{\mathrm{th}}$ and reports 1-bit information to the transmitter about the comparison. In such a way, the transmitter can partition all the users into two groups and schedule a user from the favorable group for transmission. Therefore, we propose the following steps for OIA using 1-bit feedback:

- Each transmitter sends out a reference signal.

- Each user equipment measures the channel quality using the chordal distance measure.

- Each user compares the locally measured chordal distance to a threshold. In case the measured value is smaller than the threshold, a ' 1 ' will be fed back; otherwise a ' 0 ' will be fed back.

- The transmitter will randomly select a random user whose feedback value is ' 1 ' for transmission.

A scheduling outage occurs if all users send ' 0 ' to the transmitter. In such an event, a random user among all users will be selected for transmission. To find the scheduling outage probability $P_{\text {out }}$, we first denote the cumulative density function $(\mathrm{CDF})$ of $\mathcal{D}_{i}^{k}$ by $F_{\mathcal{D}}(x)$, which is defined as

$$
\begin{aligned}
F_{\mathcal{D}}(x) & =\operatorname{Pr}\left(\mathcal{D}_{i}^{k} \leq x\right) \\
& =\operatorname{Pr}\left(d_{\mathrm{c}}^{2}\left(\mathbf{A}, \mathbf{C}_{k}\right) \leq x\right) \\
& \approx \begin{cases}0, & x<0 \\
c_{N_{\mathrm{R}}, d} \cdot x^{d\left(N_{\mathrm{R}}-d\right)}, & 0 \leq x \leq \hat{x} \\
1, & x>\hat{x}\end{cases}
\end{aligned}
$$


where $\hat{x}$ satisfies $c_{N_{\mathrm{R}}, d} \cdot \hat{x}^{d\left(N_{\mathrm{R}}-d\right)}=1$ and $\hat{x} \leq d$. If $d=1$, the CDF of (18) becomes exact. If $d>1$, the CDF in (18) is exact when $0 \leq x \leq 1$. When $1<x<d$, the $\mathrm{CDF}$ provided by (18) deviates from the true CDF [12]. However, we are mainly interested in small $x<1$ for the purpose of feedback reduction by thresholding.

Therefore, the scheduling outage probability corresponds to the event where all $K$ users exceed $x$, which is denoted by

$$
\begin{aligned}
P_{\text {out }} & =\operatorname{Pr}\left(\min _{k} \mathcal{D}_{i}^{k} \geq x\right) \\
& =\operatorname{Pr}\left(\min _{\mathbf{C}_{k} \in \mathcal{C}_{\text {rnd }}} d_{\mathrm{c}}^{2}\left(\mathbf{A}, \mathbf{C}_{k}\right) \geq x\right) \\
& =\left(1-F_{\mathcal{D}}\left(x_{\mathrm{th}}\right)\right)^{K} .
\end{aligned}
$$

We define the probability density functions (PDFs) of $\mathcal{D}_{i}^{k}$ as $f_{\mathcal{D}}(x)$, where $\int_{0}^{x} f_{\mathcal{D}}(x) \mathrm{d} x=F_{\mathcal{D}}(x)$. In order to distinguish from the previous conventional OIA, we employ $k^{\dagger}$ as the index of the selected user with 1-bit feedback. The expected metric value of the selected user $k^{\dagger}$ can be expressed as

$$
\begin{aligned}
& \mathbb{E}\left[\mathcal{D}_{i}^{k^{\dagger}}\right] \\
& =\left(1-P_{\text {out }}\right) \int_{0}^{x_{\mathrm{th}}} \frac{f_{\mathcal{D}}(x) x}{F_{\mathcal{D}}\left(x_{\mathrm{th}}\right)} \mathrm{d} x+P_{\text {out }} \int_{x_{\mathrm{th}}}^{d} \frac{f_{\mathcal{D}}(x) x}{1-F_{\mathcal{D}}\left(x_{\mathrm{th}}\right)} \mathrm{d} x,
\end{aligned}
$$

where $\frac{f_{\mathcal{D}}(x)}{F_{\mathcal{D}}\left(x_{\mathrm{th}}\right)}$ and $\frac{f_{\mathcal{D}}(x)}{1-F_{\mathcal{D}}\left(x_{\mathrm{th}}\right)}$ are the normalized truncated PDFs of $\mathcal{D}_{i}^{k}$ in the corresponding intervals $\left[0, x_{\mathrm{th}}\right)$ and $\left[x_{\mathrm{th}}, d\right]$, satisfying $\int_{0}^{x_{\mathrm{th}}} \frac{f_{\mathcal{D}}(x) \mathrm{d} x}{F_{\mathcal{D}}\left(x_{\mathrm{th}}\right)}=1$ and $\quad \int_{x_{\mathrm{th}}}^{d} \frac{f_{\mathcal{D}}(x) \mathrm{d} x}{1-F_{\mathcal{D}}\left(x_{\mathrm{th}}\right)}=1$. The first term in (22) represents the event where at least one user falls below the threshold and reports ' 1 ' to the transmitter. The second term denotes a scheduling outage, where all the users exceed the threshold and report ' 0 '.

\section{Achievable DoF and User Scaling Law When $d=1$}

For a given $K, P_{\text {out }}$ is uniquely determined by the choice of the threshold $x_{\mathrm{th}}$. We intend to find the optimal $x_{\mathrm{th}}$, such that (22) is minimized. The function is convex in the range of $[0,1]$. Thus, $\mathbb{E}\left[\mathcal{D}_{i}^{k^{\dagger}}\right]$ has an unique minimum within the interval $[0,1]$. To find the minimum value and the corresponding threshold, we need to solve the equation $\frac{\partial \mathbb{E}\left[\mathcal{D}_{i}^{k^{\dagger}}\right]}{\partial x_{\mathrm{th}}}=0$. For $d=1$, according to (18) we have $F_{\mathcal{D}}(x) \stackrel{0}{=} x$ and $f_{\mathcal{D}}(x)=1$ in the interval $[0,1]$. The expected metric value $\mathbb{E}\left[\mathcal{D}_{i}^{k^{\dagger}}\right]$ in (22) can be simplified as

$$
\begin{aligned}
D_{i}\left(x_{\mathrm{th}}\right) & =\mathbb{E}\left[\mathcal{D}_{i}^{k^{\dagger}}\right] \\
& =\left(1-P_{\mathrm{out}}\right) \int_{0}^{x_{\mathrm{th}}} \frac{x \mathrm{~d} x}{x_{\mathrm{th}}}+P_{\text {out }} \frac{\int_{x_{\mathrm{th}}}^{1} x \mathrm{~d} x}{1-x_{\mathrm{th}}} \\
& =\left(1-\left(1-x_{\mathrm{th}}\right)^{K}\right) \frac{x_{\mathrm{th}}}{2}+\left(1-x_{\mathrm{th}}\right)^{K}\left(\frac{1+x_{\mathrm{th}}}{2}\right) .
\end{aligned}
$$

The optimal $x_{\text {th }}$ which minimizes $\mathbb{E}\left[\mathcal{D}_{i}^{k^{\dagger}}\right]$ can be found by solving $\frac{\partial D_{i}\left(x_{\mathrm{th}}\right)}{\partial x_{\mathrm{th}}}=0$, i.e. $-K\left(1-x_{\mathrm{th}}\right)^{K-1}+1=0$. Thus we have the optimal threshold

$$
\hat{x}_{\mathrm{th}}=1-\left(\frac{1}{K}\right)^{\frac{1}{K-1}} \text {. }
$$

Applying $\hat{x}_{\mathrm{th}}$ to (23), the minimum of $D_{i}\left(x_{\mathrm{th}}\right)$ can be written as a function of $K$ as

$$
D_{i}\left(\hat{x}_{\mathrm{th}}\right)=\frac{1}{2}\left(\frac{1}{K}\right)^{\frac{K}{K-1}}-\frac{1}{2}\left(\frac{1}{K}\right)^{\frac{1}{K-1}}+\frac{1}{2} .
$$

This leads us to the following lemma, which will then be used for the proof of the achievable DoF.

Lemma 1. When the number of users $K$ goes to infinity, i.e. $K \rightarrow \infty, D_{i}\left(\hat{x}_{\mathrm{th}}\right)$ is asymptotically equivalent to $\frac{\log (K)}{2 K}$, such that

$$
\lim _{K \rightarrow \infty} \frac{D_{i}\left(\hat{x}_{\mathrm{th}}\right)}{\frac{\log K}{2 K}}=1 .
$$

Proof. Accroding to (25), the left hand side of (26) can be written as

$$
\begin{aligned}
& \lim _{K \rightarrow \infty} \frac{\left(\frac{1}{K}\right)^{\frac{K}{K-1}}-\left(\frac{1}{K}\right)^{\frac{1}{K-1}}+1}{\frac{\log K}{K}} \\
& =\lim _{K \rightarrow \infty} \frac{\left(\frac{1}{K}\right)-\left(\frac{1}{K}\right)^{\frac{1}{K}}+1}{\frac{\log K}{K}} \\
& =\lim _{M \rightarrow 0} \frac{M^{M}(\log M+1)-1}{\log M+1} \\
& =\lim _{M \rightarrow 0} M^{M}-\lim _{M \rightarrow 0} \frac{1}{\log M+1} \\
& =1
\end{aligned}
$$

where (29) is obtained by letting $M=1 / K$ and applying the L'Hôpital's rule. Thus, the proof is complete.

Theorem 1. For $d=1$, if the threshold is optimally chosen according to (24), 1-bit feedback per user is able to achieve a DoF $d^{\prime} \in[0,1]$ per transmitter if the number of users is scaled as

$$
K \propto P^{d^{\prime}} .
$$

Proof. The achievable DoF of transmitter $i$ using OIA can be expressed as $1-d_{\text {loss }}$. If $K \propto P^{d^{\prime}}$, the DoF loss term can be written as

$$
\begin{aligned}
d_{\mathrm{loss}} & =\lim _{P \rightarrow \infty} \frac{\mathbb{E}\left[R_{\operatorname{loss} i}^{k^{\dagger}}\right]}{\log _{2} P} \\
& \leq \lim _{P \rightarrow \infty} \frac{\log _{2}\left(1+P D_{i}\left(\hat{x}_{\mathrm{th}}\right)\right)}{\log _{2} P} \\
& =\lim _{P \rightarrow \infty} \frac{\log _{2}\left(P D_{i}\left(\hat{x}_{\mathrm{th}}\right)\right)}{\log _{2} P} \\
& =\lim _{P \rightarrow \infty} \frac{\log _{2}\left(P \cdot \frac{\log K}{2 K}\right)}{\log _{2} P} \\
& =\left(1-d^{\prime}\right)+\lim _{P \rightarrow \infty} \frac{1}{\log P+O(1)} \\
& =\left(1-d^{\prime}\right) .
\end{aligned}
$$

The inequality (33) is obtained by using the upper bound in (13) and invoking (25). Equality (35) is due to the asymptotic equivalence in Lemma 1. Equality (36) is obtained using the 


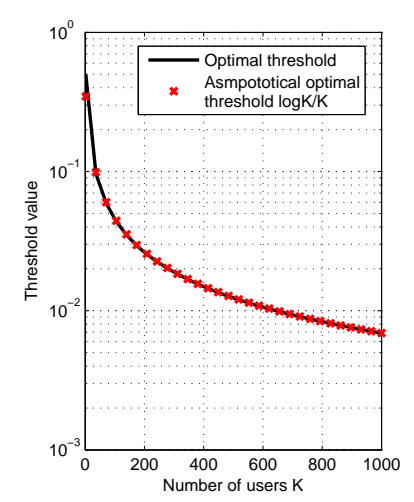

Fig. 2. Comparison among the closed-form optimal threshold and the asymptotically optimal threshold for $d=1$

relationship $K \propto P^{d^{\prime}}$ and the L'Hôpital's rule. Therefore, the DoF $d^{\prime}$ is obtained at each transmitter.

Remark 1. Compared to conventional OIA in [7], the user scaling law achieving DoF $d^{\prime}$ remains the same. The second term in (36) does not exist for conventional OIA. However, it goes to 0 when $P \rightarrow \infty$, and thus does not change the DoF. Therefore, 1-bit feedback neither degrades the performance in terms of DoF nor requires more users to achieve the same DoF.

\section{Achievable DoF and User Scaling Law When $d>1$}

For $d>1$, due to the fact that an explicit expression for $F_{\mathcal{D}}(x)$ is unknown for $x \in(1, d]$, we first derive an upper bound for (22). However, an explicit solution of $x_{\text {th }}$ which minimizes the upper bound is still intractable. For this reason, we employ asymptotic analysis and find a solution of $x_{\mathrm{th}}$, which approaches the optimal value when $K \rightarrow \infty$. Therefore, we arrive at the following theorem.

Theorem 2. If the number of users is scaled as $K \propto P^{d d^{\prime}}$, the feedback of only 1-bit per user is able to achieve the DoF $d^{\prime} \in[0, d]$ per transmitter if the threshold $\hat{x}_{\text {th }}$ is chosen such that

$$
c \hat{x}_{\mathrm{th}}^{d^{2}}=\frac{1}{K}(A \log K+B) .
$$

Proof. Due to the space limit, the proof is given in [13].

Remark 2. The result above is also applicable to the case of $d=1$. For $d=1$, the optimal threshold obtained in (24) is asymptotically equivalent to the above result.

\section{Simulation RESUlts}

In this section, we provide numerical results of the thresholds and sum rate of OIA using 1-bit feedback. Fig. 2 compares the threshold as a function of the number of users $K$ for $N_{\mathrm{R}}=2, d=1$. The thresholds are obtained by (24) and the asymptotic expression $\frac{\log K}{K}$ as mentioned in Remark 2. It can be seen that these thresholds are very close to each other, even for a small number of users $K$. Therefore, this result validates the calculations of our closed-form threshold and the asymptotic optimal threshold.

Fig. 3 shows the achievable sum rate versus SNR of OIA with full feedback and OIA with 1-bit feedback, for $N_{\mathrm{R}}=2$, $d=1$ and the number of users $K=\lceil P\rceil$. We include also the sum rate achieved by closed-form IA in 3 -user $2 \times 2$ MIMO channels. The threshold of our feedback scheme is calculated according to (24). We can see that OIA with 1-bit feedback achieves slightly lower rate than OIA with full feedback. At $30 \mathrm{~dB}$ SNR, it can achieve $85 \%$ of the sum rate obtained by full feedback OIA. Importantly, OIA with 1-bit feedback is able to capture the slope and achieve the DoF $d=1$ (see the reference line in Fig. 3).

\section{CONCLUSION}

We analyzed the achievable DoF using a 1-bit quantizer for OIA. We proved that 1-bit feedback is sufficient to achieve the optimal DoF of $d$ in 3-cell MIMO interference channels. Most importantly, the required user scaling law remains the same as for OIA with full feedback. We derived a closedform threshold for $d=1$. In the case of $d>1$, an asymptotic threshold choice was given, which is optimal when the number of users $K \rightarrow \infty$.

\section{REFERENCES}

[1] V. Cadambe and S. Jafar, "Interference Alignment and Degrees of Freedom of the K-User Interference Channel," IEEE Trans. Inf. Theory, vol. 54, no. 8, pp. 3425-3441, Aug. 2008.

[2] H. J. Yang, W.-y. Shin, B. C. Jung, and A. Paulraj, "Opportunistic interference alignment for MIMO interfering multiple-access channels," IEEE Trans. Wirel. Commun., vol. 12, no. 5, pp. 2180-2192, May 2013.

[3] B. C. Jung, D. Park, and W. Shin, "Opportunistic interference mitigation achieves optimal degrees-of-freedom in wireless multi-cell uplink networks," IEEE Trans. Commun., vol. 60, no. 7, pp. 1935-1944, Jul. 2012.

[4] T. Gou, T. Koike-Akino, and P. Orlik, "Improved and opportunistic interference alignment schemes for multi-cell interference channels," in Proc. IEEE Veh. Technol. Conf., May 2012, pp. 1-5.

[5] J. H. Lee, W. Choi, and B. D. Rao, "Multiuser diversity in interfering broadcast channels: achievable degrees of freedom and user scaling law,' IEEE Trans. Wirel. Commun., vol. 12, no. 11, pp. 5837-5849, Nov. 2013.

[6] H. J. Yang, W.-y. Shin, B. C. Jung, C. Suh, and A. Paulraj, "Opportunistic downlink interference alignment," in Proc. IEEE Int. Symp. Inf. Theory, vol. 94305, 2014, pp. 1588-1592.

[7] J. H. Lee and W. Choi, "On the achievable DoF and user scaling law of opportunistic interference alignment in 3-transmitter MIMO interference channels," IEEE Trans. Wirel. Commun., vol. 12, no. 6, pp. 2743-2753, Jun. 2013.

[8] D. Gesbert and M. Alouini, "How much feedback is multi-user diversity really worth?" in Proc. IEEE Int. Conf. Commun., 2004, pp. 234-238.

[9] M. Sharif and B. Hassibi, "On the capacity of MIMO broadcast channels with partial side information," IEEE Trans. Inf. Theory, vol. 51, no. 2, pp. 506-522, Feb. 2005.

[10] S. Sanayei and A. Nosratinia, "Opportunistic Downlink Transmission With Limited Feedback," IEEE Trans. Inf. Theory, vol. 53, no. 11, pp. 4363-4372, Nov. 2007.

[11] Z. Xu, M. Gan, and T. Zemen, "Threshold-based selective feedback for opportunistic interference alignment," in Proc. IEEE Wirel. Commun. Netw. Conf., 2015, pp. 276-280.

[12] W. Dai, Y. E. Liu, and B. Rider, "Quantization bounds on Grassmann manifolds and applications to MIMO communications," IEEE Trans. Inf. Theory, vol. 54, no. 3, pp. 1108-1123, Mar. 2008.

[13] Z. Xu, M. Gan, and T. Zemen, "On the degrees of freedom for opportunistic interference alignment with 1-bit feedback: The 3 cell case." [Online]. Available: http://arxiv.org/abs/1501.04312 